\title{
Efecto de un medio filtrante de arena común y carbón activado sobre la calidad del agua para uso de las tropas del Ejército Nacional de Colombia en el área de operaciones
}

\author{
Edgar Agudelo García \\ Dagoberto Reinoso Marroquín \\ Walter Andrés Ríos Molano \\ Sergio Andrés Blanco Londoño \\ Escuela Militar de Cadetes "General José María Córdova”
}

\section{RESUMEN}

El consumo de agua contaminada en el área de operaciones ha generado enfermedades de origen hídrico en las tropas del Ejército Nacional de Colombia (EJC). Esta investigación describe el efecto en la calidad del agua del medio filtrante de un filtro portátil de arena común y carbón activado, el cual puede ser transportado por las tropas del EJC y permitiría el consumo de agua de diferentes cuerpos de agua que puedan encontrar. Los resultados mostraron que el carbón activado fue un material adecuado para la filtración, por el contrario la arena empleada no mejoró la calidad del agua.

PALABRAS CLAVE

Agua potable; arena común; calidad del agua; carbón activado; enfermedades de origen hídrico; filtro portátil.

\section{CITACIÓN}

Agudelo García, E., Reinoso Marroquín, D., Ríos Molano, W. A., \& Blanco Londoño, S. A. (2019). Efecto de un medio filtrante de arena común y carbón activado sobre la calidad del agua para uso de las tropas del Ejército Nacional de Colombia en el área de operaciones. Revista Brújula de Investigación, 7(14), 26-30. https://doi.org/10.21830/ 23460628.17 


\section{Introducción}

El consumo de agua contaminada por desechos humanos, animales o químicos puede causar enfermedades de origen hídrico. Por ejemplo, amebiasis, cólera, diarrea, hepatitis, meningitis, entre otras (Ríos-Tobón et al., 2017). En general, la mayoría de esas enfermedades son causadas por organismos acuáticos que pasan una parte de su ciclo de vida en el agua y otra parte como parásitos de animales. Aunque estas enfermedades no son mortales, impiden a las personas llevar una vida normal y reducen su capacidad para trabajar.

En el Ejército Nacional de Colombia (EJC), las operaciones de control territorial obligan a nuestros hombres a patrullar diferentes zonas de Colombia, por lo cual no pueden cargar demasiada agua potable en sus cantimploras o en sus equipos. Por lo tanto, las tropas se ven obligadas a consumir agua de ríos, caños, quebradas y diferentes sitios donde se puede encontrar, pero de la cual desconocen su origen y calidad. Así, el consumo de agua no potable ha sido la causa principal del problema de sanidad que aqueja a las unidades del EJC (Frías-Salcedo, 2017).

La implementación de un filtro purificador puede ayudar a evitar esas enfermedades, filtrando las bacterias y organismos que contaminan el agua (Clasen et al., 2004). Los filtros de agua portátiles que son una versión sencilla de un filtro purificador están conformados por un material poroso y carbón activado (Parada et al., 2016). El funcionamiento de un filtro de agua es muy sencillo: las partículas sólidas que se encuentran en el agua se separan mediante un medio filtrante que permite el paso del fluido, pero retiene las partículas sólidas, en este caso los microorganismos patógenos principalmente (Pérez-Vidal et al., 2016). Generalmente, los materiales para elaborar un filtro de agua son arena y carbón activado (Gerba y Naranjo, et al., 2000). Sin embargo, el efecto de las características y proporciones de estos materiales sobre la calidad del agua es variable. Por lo tanto, el objetivo de esta investigación fue evaluar el efecto del medio filtrante con arena común y carbón activado de un filtro de agua portátil para incorporar al equipo de las tropas del EJC que permanecen en el área de operaciones.

\section{Materiales y métodos}

Para elaborar el filtro se utilizó una botella plástica de $250 \mathrm{~mL}$. El medio filtrante fue conformado por arena y carbón activado. También se empleó una capa de algodón en la parte inferior del medio filtrante para retener las partículas de arena y/o carbón. La arena fue pasada por un tamiz de 0,060 mm. Se empleó arena común (sucia), aquella que las tropas del EJC pueden encontrar cerca de los ríos. El carbón activado estaba en su forma bruta de granito, y presentó una estructura amorfa aleatoria altamente porosa y con poros de distintas dimensiones.

El agua cruda en estudio fue una muestra de agua del río Meta, la cual presentó gran cantidad de sedimentos y material en suspensión. Las principales características fisicoquímicas de esta muestra fueron: color 30 UPC, turbiedad 8 UNT, conductividad eléctrica $140 \mu \mathrm{S} / \mathrm{cm}, \mathrm{pH}$ 6,9 unidades, sulfatos $1,4 \mathrm{mg} / \mathrm{L} \mathrm{SO}_{4}$, cloruros 11,0 mg/L Cl y dureza 29,4 mg/L $\mathrm{CaCO}_{3}$.

El diseño experimental consistió en elaborar seis prototipos con el medio filtrante conformado por arena y carbón activado en las proporciones que se presentan en la tabla 1. Luego, se filtró una muestra del agua cruda y el efluente filtrado se recolectó en un matraz Erlenmeyer de 250 $\mathrm{mL}$. El efluente fue caracterizado en función del color, turbiedad, conductividad eléctrica (CE), $\mathrm{pH}$, sulfatos, cloruros y dureza. Todos los aná- 
lisis fueron realizados conforme lo estipula el Standard methods for the examination of water and wastewater (Eaton et al., 2005).

Tabla 1. Descripción de los prototipos

\begin{tabular}{ccc}
\hline \multirow{2}{*}{ Prototipo } & \multicolumn{2}{c}{$\begin{array}{c}\text { Conformación del medio } \\
\text { filtrante }\end{array}$} \\
\cline { 2 - 3 } & Arena (\%) & $\begin{array}{c}\text { Carbón } \\
\text { activado (\%) }\end{array}$ \\
\hline P1 & 100 & 0 \\
\hline P2 & 80 & 20 \\
\hline P3 & 60 & 40 \\
\hline P4 & 40 & 60 \\
\hline P5 & 20 & 80 \\
\hline P6 & 0 & 100 \\
\hline
\end{tabular}

Fuente: Original de los autores.

\section{Resultados y discusión}

En la figura 1 se presentan las características fisicoquímicas del agua cruda (AC) y agua filtrada (P1, P2, P3, P4, P5 y P6). También se indica mediante una línea a trazos el límite máximo aceptable de calidad del agua para consumo humano establecido por la normatividad nacional vigente, Resolución 2115 de 2007 del Ministerio de Protección Social y Ministerio de Ambiente, Vivienda y Desarrollo Territorial (2007). En las figuras 1B, 1F, 1G y $1 \mathrm{H}$ no se representa el máximo valor aceptable debido a que esos valores son muy superiores a los observados en el agua cruda. Los valores máximos según la Resolución 2115 de 2007 para los sulfatos, cloruros y dureza son $250 \mathrm{mg} / \mathrm{L} \mathrm{CaCO}_{3}, 250$ mg/L Cl y 300 mg/L CaCO ${ }_{3}$ respectivamente.

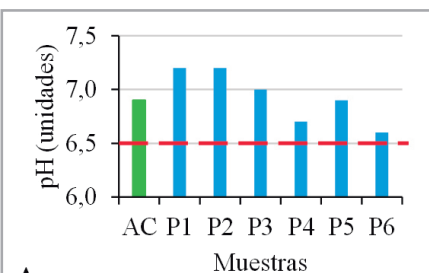

A

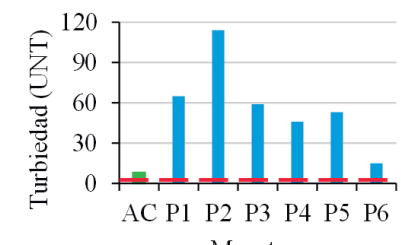

D

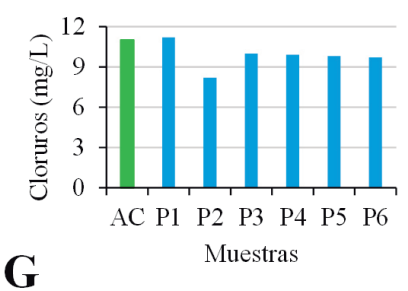

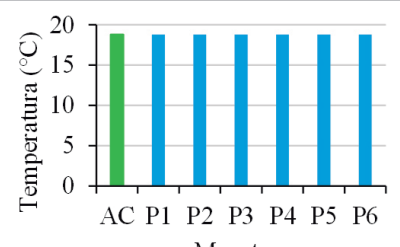

B

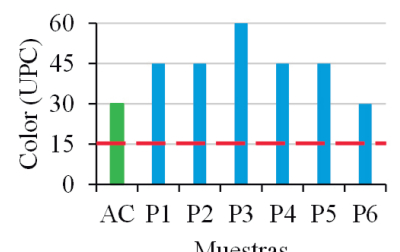

E

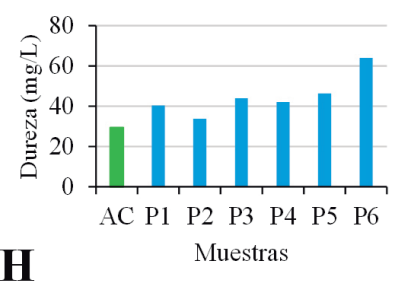

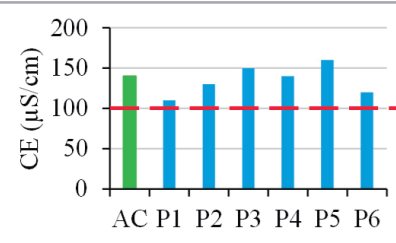

C Muestras

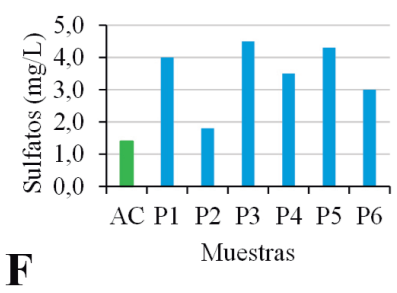

Figura 1. Características fisicoquímicas del agua cruda ( ) y agua filtrada ( ).Valor máximo aceptable de calidad del agua para consumo humano, según Resolución 2115 de 2007 (-ーー).

Fuente: Original de los autores. 
Asimismo, los resultados relacionados con la calidad fisicoquímica del agua filtrada en términos de CE (figura 1C), turbiedad (figura 1D) y color (figura 1E) no fueron satisfactorios debido a que los valores finales fueron superiores a los observados en el agua cruda, y en ningún caso, cumplieron con el estándar de calidad de agua para consumo humano estipulado en la Resolución 2115 de 2007. Probablemente las impurezas del medio filtrante fueron responsables del comportamiento observado. Debido a que la turbiedad y el color fueron las variables más alteradas en el efluente, es posible suponer que la arena contenía partículas sólidas o coloidales que al entrar en contacto con el agua fueron arrastradas a través del medio filtrante, de manera que deterioraron la calidad fisicoquímica del agua filtrada.

Un resultado interesante que se observó en esta investigación se relaciona con la capacidad del carbón activado para eliminar los cloruros. En la figura $1 \mathrm{G}$ se observa que el efluente de los prototipos que contenían carbón activado en diferentes proporciones (P2 a P6) es mejor respecto al prototipo P1 que solo contenía arena, es decir que el carbón activado es un material adecuado para la eliminación de cloruros.

\section{Conclusiones}

El desarrollo del presente trabajo permitió identificar la viabilidad de un filtro portátil con medio filtrante de arena común y carbón activado en relación con la normatividad nacional vigente de agua potable. La calidad y el tipo de arena no fueron efectivas para filtrar el agua debido a que parámetros como la turbiedad y el color aumentaron en el agua filtrada. Por el contrario, el carbón activado fue un material adecuado para la filtración. Sin embargo, para que el filtro portátil cumpla con las condiciones de agua potable, se debe experimentar con otro tipo de medios filtrantes adicionales, por ejemplo arena lavada, membranas, sílice, entre otros. La utilidad que se le puede dar al filtro portátil y al conocimiento para realizarlo en el área de operaciones disminuiría el número de tropas con enfermedades de origen hídrico.

\section{Agradecimientos}

Los autores agradecen a la Escuela Militar de Cadetes General José María Córdoba y al Laboratorio de Calidad de Aguas de la Universidad Militar Nueva Granada.

\section{Declaración de divulgación}

Los autores declaran que no existe ningún potencial conflicto de interés relacionado con el texto.

\section{Financiamiento}

Los autores no declaran fuente de financiamiento para la realización de este artículo.

\section{Sobre los autores}

Edgar Agudelo García es estudiante de la Facultad de Ingeniería Civil de la Escuela Militar de Cadetes General José María Córdova, Bogotá, D. C., Colombia.

Dagoberto Reinoso Marroquín es estudiante de la Facultad de Ingeniería Civil de la Escuela Militar de Cadetes General José María Córdova, Bogotá, D. C., Colombia.

Walter Andrés Ríos Molano es estudiante de la Facultad de Ingeniería Civil de la Escuela Militar de Cadetes General José María Córdova, Bogotá, D. C., Colombia.

Sergio Andrés Blanco Londoño es ingeniero civil y especialista en Planeación Ambiental y Manejo Integral de los Recursos Naturales de la Universidad Militar Nueva Gra- 
nada y magíster en Recursos Hidráulicos de la Universidad Nacional de Colombia, docente de la Facultad de Ingeniería Civil de la Escuela Militar de Cadetes General José María Córdova, Bogotá, D. C., Colombia. Contacto: sergio.blanco@ esmic.edu.co

\section{Referencias}

Eaton, A. D., American Public Health Association, American Water Works Association, Water Environment Federation. (2005). Standard methods for the examination of water and wastewater. Washington, D. C.: American Public Health Association.

Clasen, T. F., Brown, J., Collin, S., Suntura, O. \& Cairncross, S. (2004). Reducing diarrhea through the use of household-based ceramic water filters: a randomized, controlled trial in rural Bolivia. The American Journal of Tropical Medicine and Hygiene, 70 (6), 651-657.

Frías-Salcedo, J. A. (2017). El género Aeromonas como patógeno humano. Revista de Sanidad Militar, 58 (4), 321-323.
Gerba, C. P. \& Naranjo, J. E. (2000). Microbiological water purification without the use of chemical disinfection. Wilderness \& Environmental Medicine, 11 (1), 12-16.

Ministerio de la Protección Social y Ministerio de Ambiente, Vivienda y Desarrollo Territorial de Colombia. (2007). Resolución 2115 de 2007. Por medio de la cual se señalan características, instrumentos básicos y frecuencias del sistema de control y vigilancia para la calidad del agua para consumo humano.

Parada, J. D. C., Alarcón, R. A., Pacheco, J. F. \& Ramírez, C. (2016). Recopilación de experiencias en la potabilización del agua por medio del uso de filtros. Inventum, 11 (20), 53-60.

Pérez-Vidal, A., Díaz-Gómez, J., Salamanca-Rojas, K. L. \& Rojas-Torres, L. Y. (2016). Evaluación del tratamiento de agua para consumo humano mediante filtros Lifestraw ${ }^{\circledR}$ y Olla Cerámica. Revista de Salud Pública, (18), 275-289.

Ríos-Tobón, S., Agudelo-Cadavid, R. M. \& Gutiérrez-Builes, L. A. (2017). Patógenos e indicadores microbiológicos de calidad del agua para consumo humano. Revista Facultad Nacional de Salud Pública, 35 (2), 236-247. 